\title{
ultramicroscopy
}

Ultramicroscopy 61 (1995) 279-283

\section{Detection of fluorescence in situ hybridization on human metaphase chromosomes by near-field scanning optical microscopy}

\author{
M.H.P. Moers * , A.G.T. Ruiter, A. Jalocha, N.F. van Hulst \\ Department of Applied Physics, University of Twente, P.O. Box 217, 7500 AE Enschede, The Netherlands
}

Received 9 May 1995; accepted 17 July 1995

\begin{abstract}
Fluorescence in situ hybridization signals on human metaphase chromosomes are detected by a near-field scanning optical microscope. This makes it possible to localize and identify several fluorescently labeled genomic DNA fragments on a single chromosome with a resolution superior to traditional fluorescence microscopy. Several nucleic acid probes have been used. The hybridization signals are well resolved in the near-field fluorescence images, and the exact location of the probes can be correlated to the topography as it is afforded by the shear-force feedback.
\end{abstract}

\section{Introduction}

Fluorescence in situ hybridization (FISH), a cytochemical technique with important applications in (cancer) cytogenetics, cell biology, virology and oncology, allows the localization of genomic DNA fragments in morphologically preserved inter- and metaphase chromosomes. Hereto a nucleic acid probe is hybridized in situ with its complementary sequence in the chromosome. Detection of the DNA probes is done by labeling with fluorescent dyes in combination with high-resolution fluorescence microscopy [1-3].

One of the main items of new developments on in situ hybridization is resolution. Detection with con-

\footnotetext{
* Corresponding author.
}

ventional fluorescence microscopy limits the physical resolution to about $0.3 \mu \mathrm{m}$ because of diffraction effects. Obviously DNA resolution, i.e. the distance in basepairs (bp), is determined by the degree of condensation of DNA in the object under study. For condensed DNA in metaphase chromosomes Lichter et al. [4] have reported a $3 \mathrm{Mb}$ (mega-basepairs) resolution, while on less condensed chromatin in interphase nuclei the DNA resolution can be as good as $50 \mathrm{~kb}$ (kilo-basepairs) [5].

Both chemical and physical approaches are to be followed to improve resolution of in situ hybridization even further. Nuclear extraction techniques, for instance, result in highly extended naked DNA loops arranged around the nuclear matrix in a halo-like structure [6], making a DNA resolution of $1 \mathrm{~kb}$ feasible with fluorescence microscopy. An important disadvantage of halo preparations, as well as interphase nuclei, is that the chromatin is decondensed 
into a high degree such that only a very small part of the total DNA is investigated. Furthermore, the relation to the physical structure and function of the chromosome is lost.

The use of high resolution microscopic techniques for the detection of in situ hybridization signals is the physical approach. Gold-labeled probes can be detected by electron microscopy with a fantastic lateral resolution of about $10 \mathrm{~nm}$ [7-10], and another candidate is atomic force microscopy (AFM): Putman et al. [11] have used an AFM to demonstrate that morphological labels, such as precipitated diaminebenzidine (DAB) [12], can be visualized as pronounced bulges on top of the chromosome structure. Apart from difficulties with chromosome preparation, an important disadvantage of these techniques is the lack of multiplicity: one is not able to recognize different DNA probes on a single chromosome, in contrast to the detection of multiple colour FISH signals with fluorescence microscopy.

The use of Near-field Scanning Optical Microscopy (NSOM) promises good prospects for the detection of multiple-colour FISH signals, since NSOM links the high resolution of scanning probe techniques to the possibilities of optical microscopy. In this paper the detection of FISH signals on human metaphase chromosomes using near-field scanning optical microscopy will be demonstrated.

\section{Materials and methods}

Routine procedures [13] were followed to produce metaphase spreads from a primary peripheral blood lymphocyte culture of a healthy male donor on microscope cover slips. Two recombinant DNA's for chromosome 1 were used: p1-79 for the telomeric region of the short arm (1p36) [14], and the plasmid probe $\alpha$-spectrin [15], that hybridizes at $1 \mathrm{q} 21.3$. Biotin or digoxygenin labeling, in situ hybridization [16], and immunological detection [17] with CY-3, an orange-red fluorescent dye, were performed according to standard procedures. Only the final step in the process, embedding of the sample in antifading liquid and counterstaining with DAPI, has been omitted. The samples are dried in air to facilitate NSOM measurements.

The near-field scanning optical microscope, including shear-force feedback regulation $[18,19]$, that is used for the detection of FISH signals is mounted on top of a Zeiss Axiovert 135 TV inverted microscope [20]. This makes it possible to preselect interesting chromosomes with the high-quality conventional fluorescence microscope. The sample is locally illuminated through a small aperture at the tapered end of an adiabatically tapered optical fiber, prepared according to standard procedures [21]. About $100 \mathrm{pW}$ of green light $(521 \mathrm{~nm})$ is emitted by

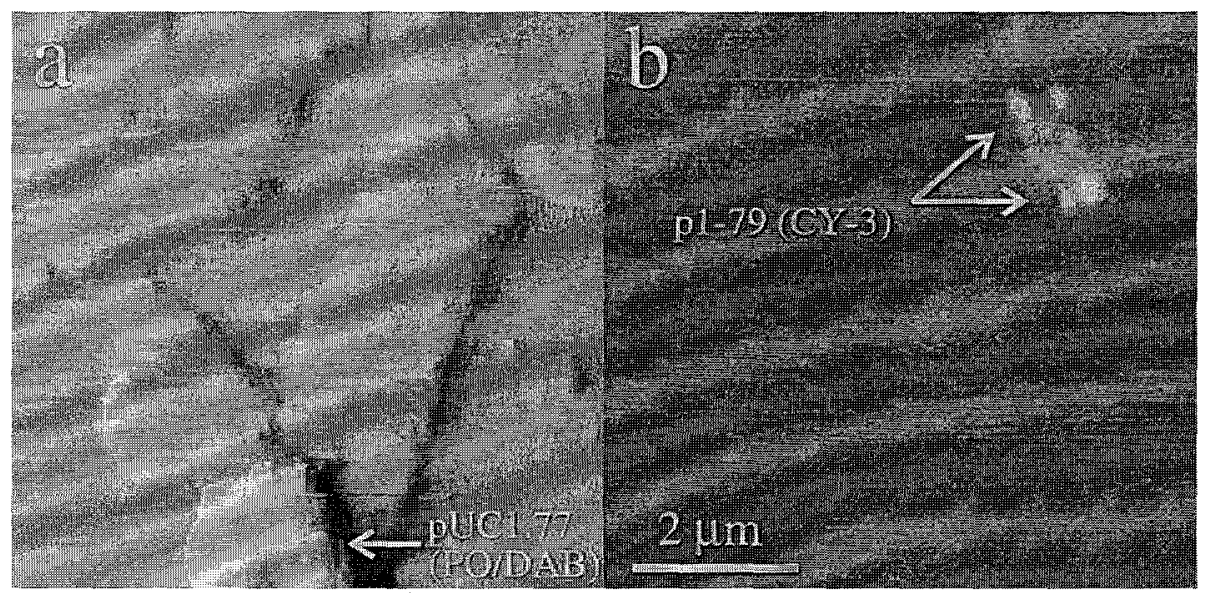

Fig. 1. A $7 \times 7 \mu \mathrm{m}^{2}$ image of human metaphase chromosomes, hybridized with pUC1.77 (PO/DAB) and p1-79 (CY-3): (a) the shear-force topographic image, (b) the fluorescence image. 


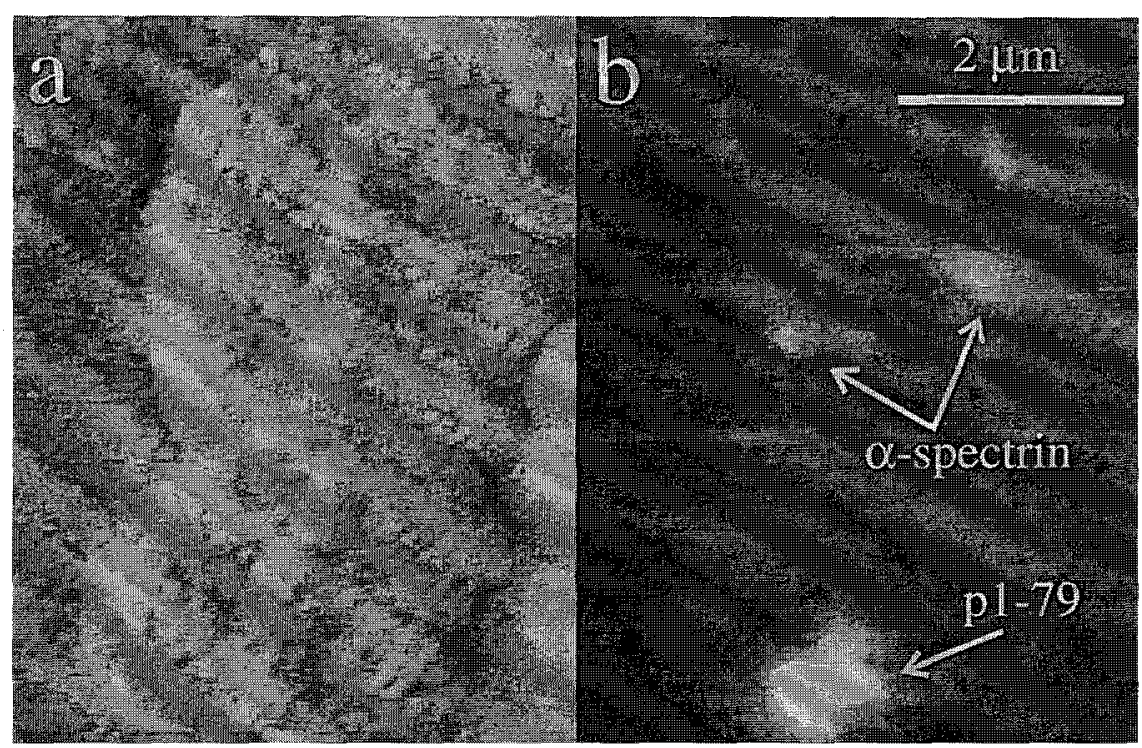

Fig. 2. A $5 \times 7 \mu \mathrm{m}^{2}$ image of a human metaphase chromosome 1 hybridized with p1-79 and $\alpha$-spectrin, both visualized with CY-3: (a) the shear-force topographic image, (b) the fluorescence image.

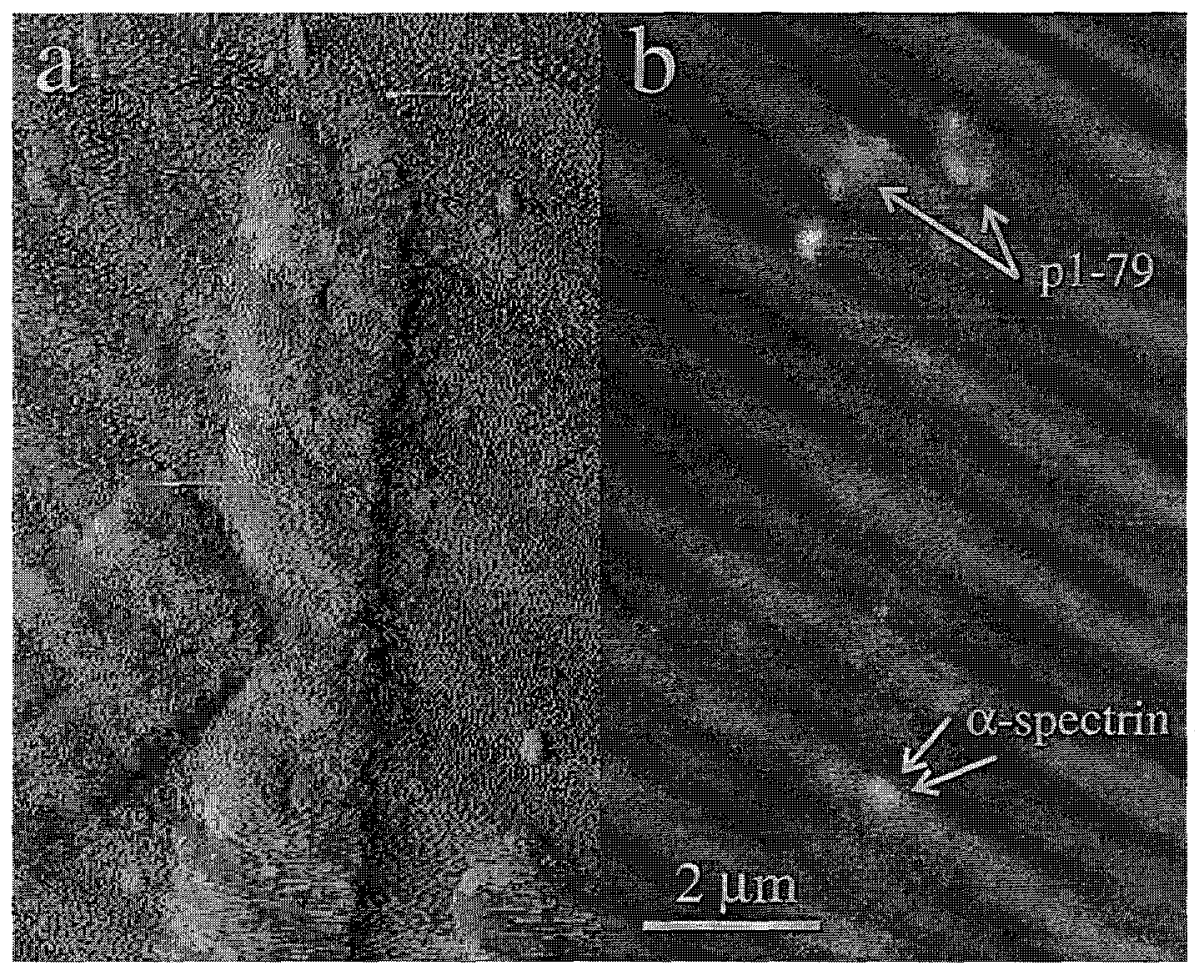

Fig. 3. A $7 \times 10 \mu \mathrm{m}^{2}$ image of a human metaphase chromosome 1 hybridized with p1-79 and $\alpha$-spectrin, both visualized with CY-3: (a) the shear-force topographic image, (b) the fluorescence image. 
the aperture. While scanning with a home-built $X Y Z$ scanner, the fluorescence is collected by a $40 \times$, 0.75 NA objective and detected by a photon counting avalanche photo-diode (EG \& G SPCM200). $\mathrm{Ar}^{+} \mathrm{Kr}^{+}$ laser light is blocked by a KV550 (Schott) optical filter, and a BG39 (Schott) filter suppresses infrared light of the shear-force laser diode.

\section{Results and discussion}

In Fig. 1a $7 \times 7 \mu \mathrm{m}^{2}$ scan of human metaphase chromosomes is shown. In the shear-force image Fig. 1a, which is slightly filtered, several chromosomes with a typical height of $100-200 \mathrm{~nm}$ are visible. Among them is chromosome 1, which is recognized by the bulge in the centromeric region. On this particular sample chromosome 1 is hybridized with $\mathrm{p} 1-79$, which is immunologically detected by the dye CY-3 $\left(\lambda_{\text {exc }}=521 \mathrm{~nm}, \lambda_{\text {em }}=550-\right.$ $600 \mathrm{~nm}$ ), as well as with the near centromeric DNA probe pUC1.77 [22], detected by the $\mathrm{PO} / \mathrm{DAB}$ reaction [12]. This reaction product shows morphological features which enables easy localization of chromosome 1 with absorption microscopy or with force microscopy [11]. In the corresponding near-field fluorescence image, Fig. 1b, the DNA probe p1-79 is clearly recognized by bright signals in the telomeric region of chromosome 1 . The probes show considerable substructure which is much better resolved than possible with standard far-field optical techniques. Apart from these signals some auto-fluorescence of the chromosomes is visible.

The ability to detect single copy genes with a NSOM is demonstrated in Figs. 2 and 3, showing two examples of a chromosome 1 co-hybridized with p1-79 and $\alpha$-spectrin, both visualized by CY -3 . The shear-force images, Fig. $2 \mathrm{a}$ and $3 \mathrm{a}$, reveal the chromosome structure, while the DNA probes are visible in the near-field fluorescent images, Fig. $2 b$ and $3 b$. The telomer probe $\mathrm{p} 1-79$, detected at the end of the short chromosome arm, again shows some sub-structure. The single copy gene $\alpha$-spectrin is usually found at the external sides of the chromatids, as in Fig. 2b, but is in some cases also detected at the internal side, as in Fig. $3 b$.

\section{Conclusion}

In this paper we have demonstrated the detection of fluorescence in situ hybridization on human metaphase chromosomes with a near-field scanning optical microscope. FISH signals are localized on the chromosomes with an optical resolution considerably better than possible with conventional optics. Simultaneously with the fluorescence micrographs, topographic images are obtained, which makes it possible to relate the location of the DNA probes to the real chromosome structure.

\section{Acknowledgements}

The authors wish to thank Wouter Kalle of the Department of Cytochemistry and Cytology of the University of Leiden in the Netherlands for performing the cytochemical part of this work. This project is financially supported by the Dutch organisation for Fundamental Research of Matter (FOM).

\section{References}

[1] G.T. Rudkin and B.D. Stollar, Nature 265 (1977) 472.

[2] J.G.J. Bauman, J. Wiegant, P. Borst and P. van Duijn, Exp. Cell Res. 128 (1980) 485.

[3] P. Lichter and D.C. Ward, Nature 345 (1990) 93.

[4] P. Lichter, C.C. Tang, K. Call, G. Hermanson, G. Evans, D. Housman and D.C. Ward, Science 247 (1990) 64.

[5] B. Trask, D. Pinkel and G. Engh, Genomics 5 (1989) 710.

[6] J. Wiegant, W. Kalle, L. Mullenders, S. Brookes, J.M.N. Hoovers, J.G. Dauwerse, G.J.B. van Ommen and A.K. Raap, Hum. Mol. Genet. 1 (1992) 587.

[7] N.J. Hutchison, P.R. Langer-Safer, D.C. Ward and B.A. Hamkalo, J. Cell Biol. 95 (1982) 609.

[8] B.A. Hamkalo, S. Narayanswami and K. Lundgren, Am. J. Anat. 185 (1989) 197.

[9] R. Fetni, R. Drouin, N. Lemieux, P.-E. Messier and C.-L. Richer, Proc. Natl. Acad. Sci. USA 88 (1991) 10916.

[10] R. Fetni, N. Lemieux, B. Malfoy, B. Dutrillaux, P.-E. Messier and C.-L. Richer, Cytogenet. Cell Genet. 60 (1992) 187.

[11] C.A.J. Putman, B.G. de Grooth, J. Wiegant, A.K. Raap, K.O. van der Werf, N.F. van Hulst and J. Greve, Cytometry 14 (1993) 356.

[12] R.C. Gaham and M.J. Karnovsky, J. Hyst. Cyt. 14 (1966) 291.

[13] J. Wiegant, N.J. Galjart, A.K. Raap and A. d'Azzo, Genomics 10 (1991) 345 . 
[14] N. Buroker, R. Bestwick, G. Haight, R.E. Magenis and M. Litt, Hum. Genet. 77 (1978) 175.

[15] L.A. Anderson, J.M. Hall, R.V. Lebo and M.C. King, Cytogenet. Cell Genet. 51 (1989) 951.

[16] J. Wiegant, T. Ried, P.M. Nederlof, M. van der Ploeg, H.J. Tanke and A.K. Raap, Nucl. Acids. Res. 19 (1991) 3237.

[17] J.G. Dauwerse, J. Wiegant, A.K. Raap, M.H. Breuning and G.J.B. van Ommen, Hum. Molec. Gen. 1 (1992) 593.
[18] R. Toledo-Crow, P.C. Yang, Y. Chen and M. Vaez-Iravani, Appl. Phys. Lett. 60 (1992) 2957.

[19] E. Betzig, P.L. Finn and J.S. Weiner, Appl. Phys. Lett. 60 (1992) 2484.

[20] M.H.P. Moers, A.G.T. Ruiter, N.F. van Hulst and B. Bölger, Ultramicroscopy 57 (1995) 289.

[21] E. Betzig and R.J. Chichester, Science 262 (1993) 1422.

[22] H.J. Cooke and J. Hindley, Nucl. Acids Res. 6 (1979) 3177. 